\title{
Intervención de Enfermería en la adolescencia: experiencia en una Institución de Estudios Secundarios Pública ${ }^{1}$
}

Daniel Martínez Esquivel ${ }^{2}$, José Alvarado Rojas ${ }^{3}$, Heilyn Campos Ramírez ${ }^{4}$, Karen Elizondo Vargas ${ }^{5}$, Hazel Esquivel Solano ${ }^{6}$, Arturo Mancía Elizondo ${ }^{7}$, Ileana Quesada Méndez ${ }^{8}$, Daniela Quirós Bermúdez ${ }^{9}$

\section{Institución: Universidad de Costa Rica}

\section{COMO CITAR}

Martinez, D.; Alvarado, J.; Campos, H.; Elizando, K.; Esquivel, H.; Mancía, A.; Quesada, I.; Quirós, D.(abril, 2013). Intervención de Enfermería en la adolescencia: experiencia en una Institución de Estudios Secuendarios Pública. Rev. Actual en Costa Rica, 24, 1-13 Recuperado de: $<$ http://www.revenf.ucr.ac.cr/saludpublica.pdf> ISSN 1409-4568

\section{RESUMEN}

La adolescencia es una etapa clave de la vida para el empoderamiento que se debe tener de la salud, sin embargo presenta muchas necesidades que aún los equipos de salud no han resuelto. En este artículo se presenta un análisis de la situación de salud de un grupo de adolescentes que asiste una institución pública de educación superior y la intervención realizada por estudiantes de Enfermería, enfocada en crear condiciones de promoción de la salud y prevención de la enfermedad. La presente investigación se desarrolló desde un enfoque cuantitativo de tipo descriptivo y fue realizada en una institución de estudios secundarios, en el período comprendido de setiembre a noviembre del año 2012. Se trabajó con alumnas y alumnos de octavo y noveno año con una muestra a conveniencia. Los principales resultados señalaron necesidades en sexualidad, hábitos de vida saludables y resolución de conflictos. Para abordar estos temas se desarrollaron distintas estrategias planificadas, organizadas, dirigidas y controladas por las personas responsables. Se concluye que la educación en salud de los y las adolescentes es un problema de salud pública que los y las enfermeras deben satisfacer.

Palabras clave: Adolescencia, Enfermería, Salud, Salud-Pública.

\footnotetext{
${ }^{1}$ Fecha de recepción: 10 de enero del 2013

Fecha de aceptación: 12 de febrero del 2013

${ }^{2}$ Enfermero. Docente, Escuela de Enfermería. Universidad de Costa Rica. Correo electrónico: dtinez@gmail.com

${ }^{3}$ Estudiante de Enfermería. Escuela de Enfermería, Universidad de Costa Rica. Correo electrónico: jbertali@hotmail.com

${ }^{4}$ Estudiante de Enfermería. Escuela de Enfermería, Universidad de Costa Rica. Correo electrónico: heylin.cr@hotmail.com

${ }^{5}$ Estudiante de Enfermería. Escuela de Enfermería, Universidad de Costa Rica. Correo electrónico: karenev91@hotmail.com

${ }^{6}$ Estudiante de Enfermería. Escuela de Enfermería, Universidad de Costa Rica. Correo electrónico: hazel.eso@gmail.com

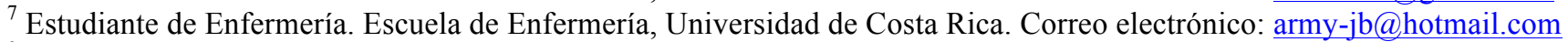

${ }^{8}$ Estudiante de Enfermería. Escuela de Enfermería, Universidad de Costa Rica. Correo electrónico: ileanaq 24@hotmail.com

${ }^{9}$ Estudiante de Enfermería. Escuela de Enfermería, Universidad de Costa Rica. Correo electrónico: dani28qb@hotmail.com
} 


\title{
Nursing Intervention in adolescence: an institution experience in Public Secondary School ${ }^{1}$
}

Daniel Martínez Esquivel ${ }^{2}$, José Alvarado Rojas ${ }^{3}$, Heilyn Campos Ramírez ${ }^{4}$, Karen Elizondo Vargas ${ }^{5}$, Hazel Esquivel Solano ${ }^{6}$, Arturo Mancía Elizondo ${ }^{7}$, Ileana Quesada Méndez ${ }^{8}$, Daniela Quirós Bermúdez ${ }^{9}$

\section{Institution: University of Costa Rica}

\section{CITED}

Martinez, D.; Alvarado, J.; Campos, H.; Elizando, K.; Esquivel, H.; Mancía, A.; Quesada, I.; Quirós, D.(abril, 2013). Nursing Intervention in adolescence: an institution experience in Public Secondary School. Rev. Actual en Costa Rica, 24, 1-13 Recuperado de:

$<$ http://www.revenf.ucr.ac.cr/saludpublica.pdf> ISSN 1409-4568

\begin{abstract}
Adolescence is a key stage of life to the empowerment that must have health, but it has many needs that health teams still have not resolved. This article presents an analysis of the health situation of a group of teenagers attending a public institution of higher education and intervention by nursing students, focused on creating conditions for health promotion and disease prevention. This research was conducted from a quantitative, descriptive and was performed in an institution of secondary education in the period from September to November 2012. We worked with students and students of eighth and ninth year with a convenience sample. The main results indicated needs in sexuality, healthy lifestyles and conflict resolution. To address these issues evolved different strategies planned, organized, directed and controlled by the individual. We conclude that health education in adolescents is a public health problem and that nurses must meet.
\end{abstract}

Key words: Adolescence, Health, Nursing, Public-health.

\footnotetext{
${ }^{1}$ Date of receipt: January 10, 2013

Date of acceptance: February 12, 2013

${ }^{2}$ Enfermero. Docente, Escuela de Enfermería. Universidad de Costa Rica. Correo electrónico: dtinez@gmail.com

${ }^{3}$ Estudiante de Enfermería. Escuela de Enfermería, Universidad de Costa Rica. Correo electrónico: jbertali@hotmail.com

${ }^{4}$ Estudiante de Enfermería. Escuela de Enfermería, Universidad de Costa Rica. Correo electrónico: heylin.cr@hotmail.com

${ }^{5}$ Estudiante de Enfermería. Escuela de Enfermería, Universidad de Costa Rica. Correo electrónico: karenev91@,hotmail.com

${ }^{6}$ Estudiante de Enfermería. Escuela de Enfermería, Universidad de Costa Rica. Correo electrónico: hazel.eso@gmail.com

${ }^{7}$ Estudiante de Enfermería. Escuela de Enfermería, Universidad de Costa Rica. Correo electrónico: army-jb@hotmail.com

${ }^{8}$ Estudiante de Enfermería. Escuela de Enfermería, Universidad de Costa Rica. Correo electrónico: ileanaq 24@hotmail.com

${ }^{9}$ Estudiante de Enfermería. Escuela de Enfermería, Universidad de Costa Rica. Correo electrónico: dani28qb@hotmail.com
} 


\section{INTRODUCCION}

La Organización Mundial de la Salud (2005), define la adolescencia como “...la etapa que transcurre entre los 11 y 19 años, considerándose dos fases, la adolescencia temprana 12 a 14 años y la adolescencia tardía 15 a 19 años", (p.4).

En cada una de las etapas se presentan cambios tanto en el aspecto fisiológico (estimulación y funcionamiento de los órganos por hormonas, femeninas y masculinas), cambios estructurales anatómicos y modificación en el perfil psicológico y de la personalidad; sin embargo la condición de la adolescencia no es uniforme y varía de acuerdo a las características individuales y de grupo.

Es importante que en cualquier abordaje e intervención que se realice con la población adolescente se tome en cuenta cada factor que influye en sus características individuales como de grupo, es por esto, que el acercamiento de la Enfermería con el y la adolescente debe hacerse de una manera integral tomando en cuenta, no solo los aspectos biológicos, sino todos aquellos elementos que influyen directamente en el pensamiento y estilo de vida del y la adolescente como es la familia, redes de apoyo, aspectos psicosociales, alimentación, sexualidad, oportunidades que le pueda ofrecer la comunidad en la que se desarrolla, accesibilidad a servicios básicos y servicios de salud, entre otros determinantes sociales que son ejes fundamentales en el desarrollo físico, psicológico y social del y la adolescente.

El Ministerio de Salud (2010), informa que existen 4 temas primordiales sobre salud en la adolescencia que se deben atender, estos temas son: salud sexual y reproductiva, salud nutricional, salud mental y violencia.

"Según la Encuesta Nacional de Salud Sexual y Reproductiva realizada en el año 2010, el $60,9 \%$ de los hombres y $49 \%$ de la mujeres de 15 a 19 años ya han tenido relaciones sexuales con penetración vaginal, la mayoría inician relaciones sexuales en edades entre 15-19 años, pero el $20,9 \%$ de los hombres reportan iniciar su primera relación sexual con penetración de 10-14 años de edad y las mujeres $9,8 \%$. Además, en ambos sexos se reporta que la primera relación sexual es con personas mayores que ellos y ellas, los hombres inician de preferencia con alguna amistad, en cambio las mujeres es con el novio". (Ministerio de Salud, 2010, (p.44)

Tomando en cuenta otro aspecto importante como es el embarazo adolescente, se reporta en las encuestas de hogares del XVI Informe del Estado de la Nación (2011), que en la población de 12 a 17 años el 15\% de las mujeres está embarazada, uno de los motivos por los cuales se da la deserción escolar.

"Se informa que el mayor número de nacimientos está en madres adolescentes aunque ha bajado un poco pasó de 20,2\% en el 2008 a 18,7\% en el 2010. En cuanto a estudiantes embarazadas de secundaria se muestra que cerca de nueve estudiantes por cada mil matriculadas están en esta condición, lo que muestra importantes déficits del sistema educativo costarricense en cuanto al objetivo estratégico de la PIEG, de ofrecer educación sexual a esta población”. (p. 110) 
Como se muestra anteriormente, las relaciones sexuales de los y las adolescentes y como consecuencia de ello, el embarazo en adolescentes, son un problema de salud pública que va en aumento, por lo cual es importante la intervención oportuna en esta población, ofreciéndoles como principal herramienta, el conocimiento por medio de una educación sexual objetiva ${ }^{1}$, en pro al bienestar de las y los adolescentes.

En cuanto al tema nutricional, según los resultados de la Encuesta Global de Salud Escolar de Costa Rica realizada en el 2009,

El sobrepeso afecta a más de una cuarta parte de las personas adolescentes encuestadas $(27,9 \%)$ y no existe una gran diferencia entre hombres y mujeres $(27,6 \%$ hombres y $28,3 \%$ mujeres $)$, mientras que la obesidad se reporta en un $8,8 \%$, afectando de una manera más significativa a los hombres con un 9,7\% y mientras que las mujeres representan un 7,9\% de la población encuestada. (Ministerio de Salud, 2010, p.7).

En cuanto a la salud mental, está íntimamente relacionada con la salud física, por lo cual aquello que nos afecte mentalmente en nuestro estado de ánimo, autoestima, autoconcepto repercutirá irremediablemente en nuestra salud física, ya sea somáticamente o bien en nuestros deseos de autocuidado, de cuidar de nuestro bienestar y salud. Respecto de lo anterior, la OPS, 2004, señala

Las estadísticas del Hospital Nacional Psiquiátrico correspondientes al mes de mayo de 2002, señalan el déficit de atención (49,8\% de los casos) como primera causa de consulta en el servicio de psiquiatría infantil y de adolescentes, seguido por la depresión (16,5\%), trastornos relacionados con el abuso sexual $(9,89 \%$ y la ansiedad $(9,41 \%)$. El $71 \%$ de las consultas correspondió a escolares, $14 \%$ a preescolares y 14,5\% a adolescentes En este mismo centro, la primera causa de egreso de menores en el año 2001 fueron los trastornos afectivos (50,32\%), ansiedad y estrés grave $(18,62 \%)$ y trastornos psicóticos $(10,7 \%)$ (OPS, 2004) p. 19)

También el ambiente o el contexto en el que se desarrollan los y las adolescentes marcan pautas de conductas que muchas veces pueden ser perjudiciales para la salud, este es el caso del consumo de drogas, determinada por influencia de los pares, por un ambiente permisivo, por factores estresantes, y decisión personal.

La educación en esta población es fundamental pues es clave para mejorar calidad de vida, sin embargo, se puede observar en el XVII Informe del Estado de la Nación (2011), existen varios factores que pueden desencadenar exclusión y deserción en los adolescentes, al respecto se señala que,

La situación socioeconómica y el contexto familiar de las y los estudiantes, en particular las condiciones de pobreza y marginalidad, la incorporación temprana al mercado laboral, la anomia familiar y las adicciones (...) Por otro lado, hay situaciones intrasistema que tornan

\footnotetext{
${ }^{1}$ Se hace referencia como educación objetiva al proceso que permite brindar información científica independiente de la manera de pensar o sentir de las personas encargadas o facilitadoras de dicha información.
} 


\section{Revista Electrónica Enfermería Actual en costa Rica}

conflictiva la permanencia de los alumnos, como la relación con los docentes y otros actores de la comunidad educativa, los métodos de enseñanza y el acceso o no a programas de apoyo, entre otros. (p. 15)

Por lo tanto, la Enfermería como ciencia y disciplina debe expandir su campo de acción más allá de lo clínico y hospitalario, es importante que se enfoque y proyecte hacia la comunidad, para que se pueda identificar de una manera más anticipada problemas de salud y así abordar con efectividad dichos problemas por medio de la promoción de la salud y prevención de la enfermedad, creando acciones enfocadas a hábitos, conductas y cuidados que no sólo recuperen la salud de una persona de manera específica, si no que supongan el mantenimiento de un ambiente comunitario seguro y saludable para todos y todas sus integrantes, teniendo en cuenta los determinantes sociales de cada población y su influencia en cada grupo etáreo, en este caso, los y las adolescentes.

El presente trabajo supone la intervención de Enfermería en la comunidad, como ente protagónico en el abordaje integral de la población adolescente de octavo y noveno año del Liceo Roberto Brenes Mesen, tomando en cuenta como ejes transversales: la sexualidad, promoción de estilos de vida saludables, relaciones interpersonales y factores sociales y psicológicos que puedan influir en la adolescencia, dicha intervención permitirá desde el punto de vista de Enfermería abordar y satisfacer las necesidades en salud que pueda presentar este sector.

El objetivo de la presente investigación fue desarrollar la intervención de Enfermería según las necesidades de salud identificadas en la población adolescente del Liceo Roberto Brenes Mesén, para esto se describieron las principales necesidades de salud encontradas mediante los instrumentos diagnósticos utilizados y se desarrollaron estrategias para su abordaje.

\section{MATERIALES Y METODO}

La presente investigación se desarrolló desde un enfoque cuantitativo de tipo descriptivo y fue realizada en una institución de educación secundaria, en el período comprendido de setiembre a noviembre del año 2012. Se trabajó con alumnas y alumnos de octavo y noveno año, en las diferentes actividades realizadas en la Institución.

Estos estudiantes fueron seleccionados debido a una recomendación realizada por parte de los encargados de la institución, ya que se consideró su posible situación de riesgo en ese momento, en cada caso se dio seguimiento al adolescente en busca de una intervención satisfactoria que cumplieran las metas establecidas en el plan de atención de Enfermería.

En el desarrollo de la investigación se utilizó el Proceso de Atención en Enfermería. Este comprende una serie de cinco fases: valoración, diagnóstico, planeamiento, ejecución del plan y evaluación (Cisneros, s.f). Para la etapa de valoración, se utilizaron estrategias como: "La caja de diagnóstico", el historial de Enfermería, valoración física y antropométrica, el APGAR familiar, el Instrumento de Tamizaje de la Caja Costarricense de Seguro Social (CCSS) y el test de Tanner, se trabajó con una muestra de 20 estudiantes de octavo año. 
Posteriormente, en la fase diagnóstica, se realizó una síntesis de la información obtenida con las actividades mencionadas anteriormente; con ello, fue posible establecer los principales diagnósticos para la población.

En la etapa de planeamiento, se diseñaron las actividades para cada situación a tratar. En la etapa de ejecución, se implementaron estrategias como: talleres sobre diferentes temas, se colocaron carteles con información de salud en el Centro Educativo, se dio consejería de Enfermería; además, se eligieron cuatro casos para ser abordados de forma individual, esto debido a su grado de complejidad. Finalmente, se realizó una evaluación del plan, en la cual se mostró que, efectivamente, los temas tratados y las actividades realizadas estuvieron de acorde con las necesidades reales de la población estudiantil, y de los cuales se obtuvieron resultados satisfactorios.

Es importante señalar, que toda la intervención se realizó mediante la implementación de varias estrategias de trabajo en el equipo de Salud para la aplicación de un nuevo modelo de atención desde la interdisciplinariedad, la participación comunitaria y la evaluación de competencias de atención integral de salud a nivel local.

\section{Consideraciones éticas}

Cada adolescente dio su consentimiento para participar en las actividades programadas, además se solicitó la autorización de cada encargado o encargada del o la adolescente para que pudiera participar del proceso. Se tomó en cuenta los principios de Bioética; autonomía, cada joven tenía la oportunidad de decidir si quería participar o no de las actividades programadas, y el equipo de salud tuvo la capacidad de respetar y ser tolerante de tales decisiones. Beneficencia, se brindó información científica de salud de acuerdo a las necesidades que se diagnosticaron, además se hizo una devolución de los resultados a las autoridades de la institución. No maleficencia, ninguno de los y las participantes puso en riesgo su integridad física, por el contrario, tanto la institución como los jóvenes, se beneficiaron de las acciones realizadas. Y justicia, los y las investigadoras participantes se caracterizaron por su alta capacidad y sensibilidad para satisfacer las necesidades diagnosticadas.

\section{RESULTADOS}

Como parte de la intervención de Enfermería con la población adolescente de una institución pública superior se realizó un diagnóstico mediante la aplicación de "La caja diagnóstica" en la cual participaron cuatro grupos de octavo año, de la cual se obtuvieron cuestionamientos relacionados en su mayoría con: sexualidad, nutrición, el proceso de desarrollo y cambios de la adolescencia, y enfermedades de transmisión sexual.

Se realizaron 20 valoraciones generales a jóvenes entre los 13 y 17 años, de alumnos de las secciones 8-5, 8-6 y 8-7; de la valoración física sobresale que los jóvenes se encuentran en buenas condiciones de desarrollo según la edad (confirmado también en el Test de Tanner), por otro lado, se encontró problemas relacionados con condiciones bucodentales como falta de higiene y ausencia de piezas dentales; en algunos casos se halló sobrepeso según las gráficas de Índice de Masa Corporal (IMC) pero al calcular el peso ideal se encontró que estaban dentro de los rangos normales para la talla y edad de los y las adolescentes.

En el historial de Enfermería; el genograma evidenció que muchos y muchas jóvenes tienen padrastro o madrastra; en cuanto a la percepción de sí mismos los y las jóvenes se describieron como personas que poseen un 
adecuado manejo del tiempo libre y recreación y cuentan con relaciones sociales. Respecto de la alimentación, se evidenció falta de información sobre nutrición ya que algunos y algunas jóvenes no desayunaban, o comían en exceso alimentos poco saludables.

En el ámbito de sexualidad sobresale el inicio de prácticas sexuales coitales a temprana edad y una marcada necesidad de información sobre el tema de infecciones de transmisión sexual (ITS) y métodos anticonceptivos. Por su parte, algunos jóvenes presentan hábitos de vida no saludables como consumo de licor y drogas, tanto licitas como ilícitas.

Del Apgar familiar se valoró que la mayoría de jóvenes refieren tener buenas relaciones familiares, confian en su familia y se comunican con ella.

Con base en lo señalado anteriormente, se elaboraron los siguientes diagnósticos de Enfermería:

- Los jóvenes adolescentes del Liceo Roberto Brenes Mesén, que participaron en la intervención de Enfermería muestran interés sobre el tema de la sexualidad, algunos y algunas han iniciado vida sexual pero necesitan información integral que abarque la afectividad, los métodos anticonceptivos como adquirir Infecciones de Transmisión Sexual o embarazos no deseados.

- Necesidad de orientación e información sobre hábitos de vida saludable, específicamente en la forma de alimentarse, de mantener una vida más activa y de evitar el consumo de sustancias dañinas para el organismo como el licor y las drogas.

- Necesitan herramientas para la resolución efectiva y asertiva de conflictos y para mantener una comunicación de calidad en sus relaciones interpersonales.

Los diagnósticos anteriores guiaron la intervención de Enfermería, mediante la aplicación de talleres sobre Sexualidad, hábitos de vida saludable, asertividad y resolución de conflictos; la consejería y propagación de carteles informativos en la institución.

\section{DISCUSIÓN}

A continuación se analizan los diagnósticos de la población que participó en la intervención de Enfermería:

Necesidad de información integral que abarque la afectividad, los métodos anticonceptivos y los riesgos de no usar protección como adquirir Infecciones de Transmisión Sexual o embarazos no deseados.

Una de las principales inquietudes que presentaron los jóvenes con quienes se intervino en el Liceo fue el tema de la sexualidad. De acuerdo a la valoración de Enfermería se trabaron tres subtemas: sexualidad y afectividad, métodos anticonceptivos e infecciones de transmisión sexual. 
Según la teoría, la iniciación tan temprana de relaciones sexuales es un factor de riesgo que puede llevar a los adolescentes a muchas complicaciones en sus vidas y salud, relacionadas con la capacidad de tomar decisiones, con embarazos no deseados, con ITS, con dificultad para terminar sus estudios. En la población adolescente una mayoría han iniciado relaciones sexuales coitales desde los 12-13 años. Con base en estos datos se consideró pertinente brindar las herramientas para que los jóvenes experimenten su sexualidad con mayor responsabilidad y conocimiento, no sólo desde el determinante biologista sino también desde la afectividad.

Según la Encuesta Global de Salud Escolar del 2009, se encuentran los siguientes datos: el 59,5\% de los y las estudiantes reportan haber utilizado condón en su primera relación sexual. Al respecto, en el nivel escolar, el mayor porcentaje de uso del preservativo es del noveno año: $54,6 \%$ en sétimo, $54,2 \%$ en octavo y $67,6 \%$ en noveno. Estos datos son significativos porque demuestran que no hay una incorporación adecuada en la mayoría de los y las adolescentes, del uso responsable del preservativo, y por ende no están teniendo conductas de autocuidado.

Con respecto a las ITS, el Departamento de Vigilancia de la Salud del Ministerio de Salud reportó para el año 2008, que el grupo de personas con edades entre los 10 y los 19 años presentó el 3\% de los casos de VIH; 7\% de los de sífilis (un total de 105 casos, 71 casos en mujeres, 34 casos de hombres); 13,2\% de de chancro; 7,8\% de los casos de uretritis sin especificar; $11,1 \%$ de gonorrea, y el 12,3\% de las enfermedades venéreas sin especificar. (Ministerio de Salud, 2010).

Para finalizar, fue muy satisfactorio impartir en las aulas un tema aún tabú en nuestra sociedad, se considera que la temática de sexualidad debe ser vista en el ámbito académico y familiar desde una perspectiva más integral, que permita a los jóvenes hablar, preguntar e informarse, para así vivir una sexualidad responsable.

\section{Necesidad de herramientas para la resolución efectiva y asertiva de conflictos y para mantener una comunicación de calidad en sus relaciones interpersonales.}

En las actividades que se realizaron en la Institución Educativa, se concluyó necesario la implementación de una actividad en la cual se abordara el tema de asertividad y resolución de conflictos, debido a la importancia de las relaciones interpersonales en la adolescencia (Moreno, Estévez, Murgui y Musitu, 2009). En esta etapa el contacto social es relevante y forma parte del desarrollo de aspectos importantes de su personalidad, que se van construyendo a lo largo del ciclo vital (Moreno et al, 2009).

Los y las jóvenes requieren herramientas que les permitan relacionarse con sus pares, de forma que esas interacciones generen fomento del autoestima, y se sientan satisfechos con su vida (Moreno et al, 2009). En el taller realizado sobre este tema, se planteó como objetivo desarrollar conceptos como asertividad, resolución de conflictos, autoestima y tipos de violencia.

La actividad se estructuró para trabajar aspectos en los que los y las adolescentes visualizaran la importancia del autoestima, como parte fundamental de su proceso de crecimiento y desarrollo personal; además, la transcendencia que posee tener en cuenta las cualidades que tienen las personas que los rodean, así como sus 


\section{Revista Electrónica Enfermería Actual en costa Rica}

defectos. Con esto se pretendió lograr que los y las jóvenes implementaran esta información en sus relaciones interpersonales, con el fin de que sean asertivas y se logren evitar conflictos y violencia, de distintos tipos, en esta población estudiantil.

En el taller se abordaron temas como qué es la asertividad, la empatía, la afectividad y el autoestima. Esta necesidad detectada responde a la iniciativa mundial de la OMS sobre las habilidades para la vida que son "habilidades o destrezas psicosociales que le facilitan a las personas enfrentarse con éxito a las exigencias y desafíos de la vida diaria." (Mantilla, 2001, p. 7). Se trata de habilidades que son necesarias para la buena convivencia y armonía con los demás, tales como: conocimiento de sí mismo, comunicación efectiva, toma de decisiones, manejo de sentimientos y emociones, empatía, relaciones interpersonales, solución de problemas y conflictos, entre otros aspectos. (Mantilla, 2001).

En este caso las habilidades fomentadas fueron la de toma de decisiones al brindarles herramientas de asertividad, con esto a su vez se trató la comunicación afectiva. Cuando se trató la afectividad y autoestima se pudo reflexionar positivamente sobre cualidades de cada uno. La empatía los hizo tomar consciencia de las consecuencias de su forma de ser con los demás. Todas estas habilidades están íntimamente relacionadas con el desarrollo personal y su desempeño con buenas relaciones interpersonales.

Se realizaron prácticas con los y las estudiantes para resolver conflictos donde se pudo observar las formas inadecuadas para solucionar los problemas (violencia física y verbal). No obstante, durante este ejercicio los y las estudiantes demostraran tener el conocimiento para abordar los conflictos, por ejemplo: el diálogo, el no uso de la violencia, la claridad y tranquilidad para expresarse. Sin embargo, muchos (as) prefieren seguir sus emociones del momento (enojo, ira) y dejarse llevar. Se aclaró la importancia de poder expresar los sentimientos y los pensamientos con serenidad, siempre y cuando se mantenga el respeto hacia los demás.

Relacionado con el tema de afectividad o autoestima, se realizó la actividad de abrazos, la cual fue emotiva y de mucho agrado para los y las jóvenes, muchos pudieron expresar su cariño sin problemas más hubieron otras personas a las cuales se le dificultó mucho realizarlo e incluso no lo hacían, pues decían no tener buenas relaciones entre ellos (as). Esto demuestra, el problema que existe en este grupo de adolescentes para expresar sus sentimientos, incluso con sus propios amigos (as).

Acerca del abordaje del tema de violencia, muchas personas no sabían exactamente qué era la violencia, ni los tipos que existían. Relacionado con lo anterior, en la actividad de resolución de conflictos muchos indirectamente manifestaron usar tipos de violencia como verbal, o física con los demás. Este es un tema en aumento, la violencia en adolescentes es un problema al que hay que tratar con prontitud. De acuerdo con lo anterior el Departamento de Análisis Estadístico de la Dirección de Planificación Institucional del Ministerio de Educación Pública en el Boletín 07-08, dice que "la cantidad de casos de violencia entre estudiantes y docentes atendidos por el personal administrativo en III Ciclo y Educación Diversificada, presenta un crecimiento desde el año 2004 (964 casos), para el curso lectivo 2007 se dio la cifra mayor (2.026 casos)." (Fallas, s.f). Al respecto el Consejo Nacional de la Política Pública de la Persona Joven realizó la primera encuesta a la población joven en el 2008, donde se reportan datos de violencia intrafamiliar: "el tema de las humillaciones, insultos, gritos o amenazas llega a obtener una cifra superior al 10\%, correspondiente a 28,076 jóvenes.” (p. 33) Los otros tipos de 
violencia física, o abuso sexual son menos del $6 \%$. Recordemos que la violencia genera violencia y lo visto y practicado en el hogar muchas veces son repetidos en los otros escenarios tales como el colegio.

\section{Necesidad de orientación e información sobre hábitos de vida saludable, específicamente en la forma de alimentarse, de mantener una vida más activa y de evitar el consumo de sustancias dañinas para el organismo como el licor y las drogas.}

Tomando en cuenta las necesidades de información que presentan los y las estudiantes en cuanto a estilos de vida saludables, fue necesaria la implementación de un taller para los grados de octavo y noveno año que abarcara los temas de nutrición, actividad física y deporte, así como uso y abuso de sustancias adictivas, esto con el fin de incrementar sus conocimientos con respecto a estos temas para prevenir acciones que pongan en riesgo su salud física y mental.

Según la última Encuesta Nacional de Nutrición del periodo 2008-2009, el 76,2\% de la población con edades comprendidas entre los 13 y 19 años presenta un estado nutricional normal, sin embargo, uno de los mayores problemas de salud corresponde al sobrepeso y la obesidad que se presentan en un $20,8 \%$ de los adolescentes tomados en cuenta para la encuesta, y afecta principalmente a las jóvenes ya que se presenta proporcionalmente más en las mujeres $(23,9 \%)$ que en los hombres $(17,4 \%)$.

Es evidente entonces como la educación nutricional es de suma importancia para que el y la adolescente pueda introyectar hábitos alimentarios que le permitan tener un desarrollo y proceso de crecimiento adecuados, por lo cual es vital explicar a los y las jóvenes los requerimientos nutricionales de micronutrientes y macronutrientes que deben incluir en su dieta diaria para que esta logre satisfacer de una manera adecuada las necesidades nutricionales del cuerpo.

Durante el taller se brindó información mediante el círculo de los alimentos para Costa Rica, en donde de manera interactiva el o la estudiante de Enfermería en conjunto con los y las adolescentes iban realizando la adecuada distribución de los alimentos. Además de ello, se proyectaron durante el taller diferentes diapositivas con información acerca de trastornos alimenticios, en la cual se logró concretar la diferencia entre bulimia y anorexia, así como las implicaciones y consecuencias que traen consigo dichos trastornos.

Respecto de la actividad física y deporte, ésta va indudablemente ligada a los problemas de peso que presenta la población en estudio. Si el o la adolescente lleva una vida de sedentarismo sumada a la mala práctica de hábitos nutricionales, las probabilidades de sufrir sobrepeso y sus consecuencias, aumentan considerablemente, es por esta razón que se considera necesario el fomento de la actividad física y el deporte en la población adolescente, de manera que se prevengan condiciones de obesidad y sobrepeso entre los y las jóvenes, al mismo tiempo que se incentive la práctica del deporte, el cual ofrece beneficios a nivel social y de relaciones interpersonales además de mejorar las condiciones físicas de la persona.

En cuanto al tema de actividad física y deporte, se logró explicar la importancia de ambos para los adecuados procesos de desarrollo de la población adolescente, para ello se realizó la dinámica llamada "construyo un cuerpo saludable" en donde los y las adolescentes divididos en diferentes grupos debían armar partes de un cuerpo construido en papel, al armar completamente el cuerpo formaban a la vez un texto que contenía datos 
importantes acerca de actividad física y deporte. Al finalizar esta actividad se realizó una pequeña charla en donde se explicaron aspectos claves acerca de la importancia de la práctica del deporte y actividad física.

Por otra parte, El IAFA (2010) establece que en Costa Rica el consumo de tabaco y alcohol suele ocurrir en la adolescencia. La edad promedio de inicio del consumo de tabaco es de 12,6 años y de 12,9 años en el caso del alcohol. Esta edad tan temprana refiere que "existe mayor probabilidad de desarrollar patrones abusivos de consumo" (Ministerio de Salud, 2010, p. 23)

También se anota que,

Sobre el uso de drogas ilícitas se tiene también porcentajes significativos donde igualmente hay un consumo considerable en población menor de edad: en el caso de drogas ilícitas, un estudio realizado en 15 cantones del Área Metropolitana, indica que el Cantón Central presenta la mayor cantidad de decomisos de drogas a personas menores de edad (53\%). De la población estudiada, el $96 \%$ de los jóvenes a los que se les decomisó droga eran de nacionalidad costarricense y el 90\% del sexo masculino; un 94\% de ellos tenían edades comprendidas entre los 14 y 17 años. En cuanto al tipo de droga decomisada en esta población, el 80,24\% correspondieron a marihuana, seguida por el crack (11,78\%). (Ministerio de Salud, 2010, pp. 24-25)

Es por esto que la intervención de Enfermería hacia los jóvenes con respecto al tema de sustancias ilícitas es vital para la prevención de acciones como las descritas en los párrafos anteriores, donde el consumo y tráfico de drogas genera problemas aún mayores, como lo son la violencia, la pobreza y la muerte, razón por la cual se busca brindar información a la población estudiantil de manera que cuenten con las herramientas para enfrentarse a una sociedad donde el tráfico de sustancias licitas e ilícitas no pasa desapercibido pero donde los jóvenes -con el conocimiento adecuado- son capaces de tomar decisiones asertivas y beneficiosas para su salud.

Durante el taller se trató de poner en evidencia las implicaciones y riesgos que trae consigo el uso y abuso de sustancias psicoactivas; para ello se presentó a los y las adolescentes un video reflexivo el cual mostraba diferentes testimonios de personas que se vieron envueltas en el problema de las drogas, luego de proyectar el video los y las jóvenes compartieron ideas o comentarios acerca de éste. De igual manera que en los temas anteriores, se logró brindar información mediante una charla participativa la cual fue apoyada con diapositivas informativas.

\section{CONCLUSIONES}

Existe una importante necesidad de educación en salud en los y las adolescentes, principalmente en temas como sexualidad y nutrición, por lo cual el abordaje realizado estuvo lejos de mitos y tabúes, para poder dar una intervención adecuada y proporcionar herramientas útiles a la población adolescente.

En relación al tema de drogas, la población adolescente, es vulnerable a esta problemática social, ya que gran parte de la población con la que se trabajó, reconoció haber probado al menos una vez algún tipo de droga, ya fuera licita o ilícita. 
En cuanto al aspecto de relaciones interpersonales y manejo de conflictos se evidenció que las actividades realizadas con los y las estudiantes como los talleres permitieron sensibilizar a la población con la que se trabajó sobre la importancia de ser asertivos para la resolución de conflictos, aportando herramientas útiles para su desarrollo como personas.

\section{REFERENCIAS}

Báez, J. (2007). Investigación cualitativa. Madrid: ESIC Editorial.

Cisneros, F. (s.f). Proceso de Atención en Enfermería (PAE). Recuperado de:

http://artemisa.unicauca.edu.co/ pivalencia/archivos/ProcesoDeAtencionDeEnfermeria-PAE.pdf

Consejo Nacional de la Política Pública de la Persona Joven. (2008). Primera Encuesta Nacional de Juventud, Costa Rica. Observatorio de la Persona Joven. San José, Costa Rica: Fondo de Población de las Naciones

Fallas, D. (s.f). Violencia Adolescente en los Centros de Educación de Costa Rica:Una explicación integral del "fenómeno". Recuperado de: http://www.psicologodouglas.com/violencia-adolescente-en-los-centros-de educacioacuten-en-costa-rica.html

Fonseca, M., Maldonado, A., Pardo, L., y Soto, M. (2007). Adolescencia, estilos de vida y promocion de hábitos saludables. Colombia: Fundación Universtitaria Manuela Betrán.

Freyre, E. (2004). La sexualidad del adolescente y problemas asociados. Perú: Academia Nacional de Medicina. Recuperado http://www.acadnacmedicina.org.pe/publicaciones/anal_agodic04/01trabajos_incorporacion/Eleodoro_Freyre_Ro man.pdf

García, A., y Carrasco, M. (2006). Diferencias de Género en el Uso de Las Drogas. Madrid: Universidad Pontifica Comillas.

Giró, J. (2007). Adolescentes: Ocio y consumo de alcohol. Madrid: Entimema.

Hernández, R., Fernández, C. y Baptista, P. (2010). Metodología de la investigación. 5ta ed. México, DF: McGraw-Hill/Interamericana Editores.

Instituto Nacional de Estadística y Censos INEC (2011). X Censo Nacional de Población y VI de Vivienda: Resultados Generales. Costa Rica: INEC. Recuperado de:http://www.inec.go.cr/A/MS/Censos/Censo\%202011/Cifras\%20preliminares/04.\%20Resultados\%20Generales $\% 20$ Censo\%202011.pdf

Mantilla, L. (2001). Habilidades para la vida: Una propuesta educativa para la promoción del desarrollo humano y la prevención de problemas psicosociales. Colombia: Fe y Alegría. 
Ministerio de Educación Pública. (2012). Programas de estudio de "Educación para la afectividad y la sexualidad integral". Costa Rica: Ministerio de Educación Pública. Recuperado de: http://www.nacion.com/MMediaFiles/nacioncom/ba/baleb636-2b95-4d9e-b551-a7c0eaa1880e.pdf

Ministerio de Salud (2010). Plan estratégico nacional de salud de las personas adolescentes 2010-2018. Costa Rica: Ministerio de Salud.

Moreno, D., Estévez, E., Murgui, S. y Musitu, G. (2009). Reputación social y violencia relacional en adolescentes: el rol de la soledad, la autoestima y la satisfacción vital. Revista anual de Psicología, 21 (4), 45-52.

Moldenhauer, N. y Ortega, M. (2004). Adolescencia \& Sexualidad. Chile: Universidad Autral de Chile. Recuperado de: http://medicina.uach.cl/saludpublica/diplomado/contenido/trabajos/1/Puerto\%20Montt\%202004/Adolescencia_y_ Sexualidad.pdf

Ortega, R. y Requejo, A. (2000). Nutriguía: manual de nutrición clínica en atención primaria. España: Editorial Complutense.

Programa Estado de la Nación. (2011). Capítulo 2: Equidad e integración social. Decimoséptimo Informe Estado de la Nación en Desarrollo Humano Sostenible. Costa Rica: Gobierno de Costa Rica. Recuperado de: http://www.estadonacion.or.cr/images/stories/informes/017/cap_2_equidad.pdf

Redondo, C., Galdó, G. y García, M. (2008). Atención al adolescente. España: Universidad de Cantabria.

Salud, O. P. (2007). Renovación de la atención primaria de salud en las Américas: documento de posición de la Organización Panamericana de Salud/Organización Mundial de la Salud (OPS/OMS). Washington, D.C: OPS.

Salud, O. P. (2008). Sistemas de salud basados en la Atención Primaria de Salud: Estrategias para el desarrollo de los equipos de APS. Washington, D.C.: OPS.

Vásquez, C., De cos Blanco, A. y López, C. (2005). Alimentación y nutrición: manual teórico-práctico. México: Ediciones Díaz de Santos.

Wilkinson, R., \& Marmot, M. (2003). Determinantes Sociales de la Salud: Los Hechos Irrefutables. Colombia: OPS. 